\title{
ODF Maxima Extraction in Spherical Harmonic Representation via Analytical Search Space Reduction
}

\author{
Iman Aganj ${ }^{1}$, Christophe Lenglet ${ }^{1,2}$, and Guillermo Sapiro ${ }^{1}$ \\ ${ }^{1}$ Department of Electrical and Computer Engineering, University of Minnesota, USA \\ ${ }^{2}$ Center for Magnetic Resonance Research, University of Minnesota, USA \\ \{iman, clenglet, guille\}@umn.edu
}

\begin{abstract}
By revealing complex fiber structure through the orientation distribution function (ODF), q-ball imaging has recently become a popular reconstruction technique in diffusion-weighted MRI. In this paper, we propose an analytical dimension reduction approach to ODF maxima extraction. We show that by expressing the ODF, or any antipodally symmetric spherical function, in the common fourth order real and symmetric spherical harmonic basis, the maxima of the two-dimensional ODF lie on an analytically derived one-dimensional space, from which we can detect the ODF maxima. This method reduces the computational complexity of the maxima detection, without compromising the accuracy. We demonstrate the performance of our technique on both artificial and human brain data.
\end{abstract}

\section{Introduction}

Diffusion-weighted MRI significantly extends the scope of the information obtained from MRI, from being solely spatially dependent to being defined on the spatialorientational domain. Fiber microstructure and orientation are inferred using this modality from the locally measured diffusion profile of water molecules. Diffusion tensor imaging (DTI) [1] effectively models the diffusion in single-fiber voxels as a Gaussian represented by its covariance tensor. As for more complex fiber architecture, q-ball imaging (QBI) [2]-[6] has been very successful in revealing intravoxel fiber orientations by introducing the orientation distribution function (ODF) as the probability of diffusion in a given direction.

Contrary to DTI, where the principal diffusion direction can be readily computed as the major eigenvector of the diffusion tensor, QBI provides a continuous spherical function which, although clearly illustrates the major diffusion orientations as its maxima, does not directly quantify them. Diffusion directions as vectors carry less information than the ODF itself does. On the other hand, their easy interpretation and their application in tractography, e.g., [7]-[9], make the ODF maxima extraction an important post-processing step still to be carefully addressed. The number of peaks can also be interpreted as a measure of white matter complexity. In addition, unlike mixture models that calculate fiber directions by describing the diffusion signal as the sum of finite discrete unidirectional components, ODF maxima are computed without any assumptions about the existence of such components. 
Exhaustive search via finite difference method has been exploited in the literature as a straightforward approach to ODF maxima extraction [3,10]. This generally requires a two-dimensional (2D) discretization of the unit sphere, resulting in computational complexity that grows quadratically with the desired resolution. Numerical optimization approaches such as gradient ascent [11], Newton-Raphson techniques [12], and Powell's method [13], have also been employed. These techniques require a guarantee of convergence and careful initialization to obtain all the maxima. Lastly, polynomial based approaches, [14]-[16], have been proposed to extract the maxima as a subset of the stationary points of the ODF. These methods exploit a transformation of the real and symmetric spherical harmonic (RSSH) basis (most efficient for ODF reconstruction [3]), to the constrained symmetric tensor or constrained homogenous polynomial bases, resulting in polynomial equations which are solved numerically.

In this paper, we propose a polynomial based approach to reduce the problem of ODF maxima extraction in the fourth order RSSH basis, from a $2 \mathrm{D}$ search on the sphere, to a one-dimensional (1D) one on an analytically-derived curve. Compared to the $2 \mathrm{D}$ problem, this approach significantly reduces the computational complexity of the search for the maxima of the ODF - or any antipodally symmetric spherical function - without compromising the precision. Contrary to [14]-[16], our method works directly in the RSSH basis and does not require the extra step of transforming the RSSH coefficients to other tensor-based bases. We suggest a discretization scheme for the 1D exhaustive search, and show experimental results on both artificial and human brain data.

We start Sec. 2 with a brief review of the RSSH basis, and continue by describing our mathematical derivation. Experimental results are presented in Sec. 3.

\section{Methods}

\subsection{ODF in Real and Symmetric Spherical Harmonic Basis}

In this work, we use the estimator derived in [6] to compute the ODF in constant solid angle (CSA). The original definition of the QBI ODF [2] does not include the Jacobian factor $r^{2}$, thereby creating the need for normalization and artificial sharpening. In contrast, the estimator in [6] is normalized, dimensionless, and has been shown to preserve the natural sharpness of the ODF.

The spherical harmonic basis is commonly used for representing spherical functions such as the ODF, allowing for sampling in any desired direction. Orthonormal spherical harmonic functions are given by

$$
Y_{l}^{m}(\theta, \phi)=\sqrt{\frac{2 l+1}{4 \pi} \frac{(l-m) !}{(l+m) !}} P_{l}^{m}(\cos \theta) e^{i m \phi},
$$

where $P_{l}^{m}(\cdot)$ is the associated Legendre function, and $\theta$ and $\phi$ are standard spherical coordinates. The assumption of the ODF being real and antipodally symmetric, 
however, makes the use of the RSSH basis [3] more suitable. RSSH functions are indexed by a single parameter $j=l(l+1) / 2+m+1$, corresponding to $l_{j}$ and $m_{j}$, as follows [3]:

$$
Y_{j}=\left\{\begin{array}{cc}
(-1)^{m_{j} \sqrt{2}} \operatorname{Re}\left\{Y_{l_{j}}^{-m_{j}}\right\}, & -l_{j} \leq m_{j}<0 \\
Y_{l_{j^{\prime}}}^{0}, m_{j}=0 \\
\sqrt{2} \operatorname{Im}\left\{Y_{l_{j}}^{m_{j}}\right\}, & 0<m_{j} \leq l_{j} .
\end{array}\right.
$$

The ODF can be computed in this basis first by using a minimum square scheme to approximate the signal, and then by analytically computing the Funk-Radon transform, [2], following the method introduced in [3]-[5] for the original QBI, and subsequently adapted in [6] for the CSA-QBI.

\subsection{ODF Maxima Extraction}

RSSH functions, being smooth, allow us to find all the local maxima of the ODF $\psi(\theta, \phi)$ as points satisfying the following properties (subscripts indicate partial derivatives):

$$
\begin{gathered}
\psi_{\theta}(\theta, \phi)=0, \\
\psi_{\phi}(\theta, \phi)=0, \\
\operatorname{det}(H(\theta, \phi)) \geq 0, \\
\operatorname{tr}(H(\theta, \phi)) \leq 0,
\end{gathered}
$$

with the Hessian matrix $H(\theta, \phi)$ defined as

$$
H(\theta, \phi)=\left(\begin{array}{ll}
\psi_{\theta \theta}(\theta, \phi) & \psi_{\theta \phi}(\theta, \phi) \\
\psi_{\theta \phi}(\theta, \phi) & \psi_{\phi \phi}(\theta, \phi)
\end{array}\right) .
$$

Equations (3) and (4) guarantee that $(\theta, \phi)$ is either an extremum or a saddle point of the ODF. Inequalities (5) and (6) filter out, respectively, the saddle points and the local minima (including possible negative lobes), leaving us only with the local maxima of the ODF. The above expressions can all be analytically computed for an ODF expressed in the RSSH basis. However, the main challenge is to find the points that simultaneously satisfy equations (3) and (4). Once they are identified, applying inequalities (5) and (6) to filter out undesired points is trivial.

Iterative approaches (e.g., Newton method) may be applied to solve equations (3) and (4). Yet, being quite sensitive to the initialization, they are not guaranteed to converge to all the maximum points. Alternatively, an exhaustive search will result in all the maxima with an accuracy determined by the discretization resolution. Nonetheless, with the ODF being a 2D manifold, the search space, and consequently the computational complexity of the algorithm, grows quadratically with the desired resolution. 
We will next show how the fourth order RSSH basis makes it possible to confine the search to a $1 \mathrm{D}$ space, thereby creating an efficient method to extract the maxima.

\subsection{Reducing the Dimension of the Search Space}

Let us assume that the ODF has been approximated in the fourth order RSSH basis, as

$$
\psi(\theta, \phi)=\sum_{j=1}^{15} a_{j} Y_{j}(\theta, \phi) .
$$

Combining equations (1), (2), and (8), while substituting the values of $P_{l}^{m}(\cos \theta)$ from Table 1 leads to

$$
\begin{aligned}
\psi(\theta, \phi)=\frac{a_{1}}{2 \sqrt{\pi}}+ & A\left(3 \cos ^{2} \theta-1\right)+B(\phi) \sin \theta \cos \theta+C(\phi) \sin ^{2} \theta \\
& +D\left(35 \cos ^{4} \theta-30 \cos ^{2} \theta+3\right) \\
& +E(\phi)\left(7 \cos ^{2} \theta-3\right) \sin \theta \cos \theta \\
& +F(\phi)\left(7 \cos ^{2} \theta-1\right) \sin ^{2} \theta+G(\phi) \cos \theta \sin ^{3} \theta \\
& +H(\phi) \sin ^{4} \theta
\end{aligned}
$$

where $A=\sqrt{\frac{5}{16 \pi}} a_{4}, B(\phi)=\sqrt{\frac{15}{8 \pi}}\left(a_{3} \cos \phi-a_{5} \sin \phi\right)$, etc. (We drop the notation $(\phi)$ in the rest of this subsection.)

We now attempt to solve Eq. (4) by deriving Eq. (9) with respect to $\phi$. We then divide it by $\sin \theta \cos ^{3} \theta$ and rearrange it, while using the identity $\sec ^{2} \theta=1+$ $\tan ^{2} \theta$, to obtain

$$
\begin{gathered}
(H+C-F)_{\phi} \tan ^{3} \theta+(G+B-3 E)_{\phi} \tan ^{2} \theta+(6 F+C)_{\phi} \tan \theta \\
+(B+4 E)_{\phi}=0
\end{gathered}
$$

Equation (10) is a cubic function of $\tan \theta$, and can be analytically solved, leading to a closed-form expression for $\theta(\phi) .{ }^{1}$ Thus, for each given $\phi$, we obtain one, two, or three different real values for $\theta$ which satisfy Eq. (4).

The curve characterized by the pair $(\theta(\phi), \phi)$ (Fig. 1(b)) is in fact our new 1D search space which contains all the ODF maxima as points satisfying equations (3), (5), and (6) (Fig. 1(c\&d)). The number of these maxima does not need to be initially specified, since it is automatically determined by the algorithm and depends on various factors, such as the number of real solutions to Eq. (10). This is particularly important in practice, as different regions of the white matter naturally exhibit different complexity. The maxima can be found using a 1D exhaustive search (see Sec. 2.4), which is considerably faster than exploring the entire $2 \mathrm{D}$ manifold of the ODF. ${ }^{2}$

\footnotetext{
${ }^{1}$ Each solution of $\tan \theta$ corresponds to a unique value of $\theta \in[0, \pi)$. Please note that this approach can also be applied in the RSSH basis of higher orders, with the difference that there may be no analytical solution for $\theta(\phi)$, and numerical methods may need to be applied.

${ }^{2}$ Such 1D exhaustive searches can also be performed using tensor-based approaches [14]-[16].
} 


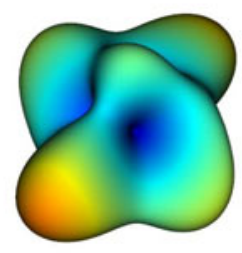

(a)

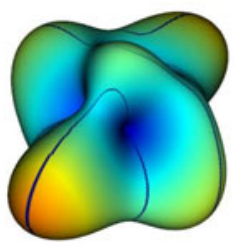

(b)

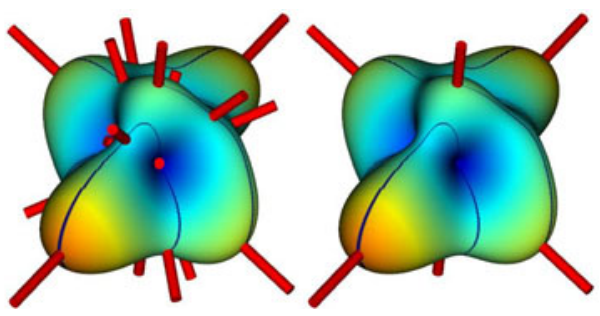

(c)

(d)

Fig. 1. (a) Reconstructed ODF. (b) Analytically defined 1D space is searched. (c) All the extrema and saddle points are identified. (d) ODF maxima are extracted.

Table 1. The associated Legendre functions required for the proposed algorithm

\begin{tabular}{ccc}
\hline Function & & Expression \\
\hline$P_{0}^{0}(\cos \theta)$ & $=$ & 1 \\
\hline$P_{2}^{0}(\cos \theta)$ & $=$ & $(1 / 2)\left(3 \cos ^{2} \theta-1\right)$ \\
$P_{2}^{1}(\cos \theta)$ & $=$ & $-3 \cos \theta \sin \theta$ \\
$P_{2}^{2}(\cos \theta)$ & $=$ & $3 \sin ^{2} \theta$ \\
\hline$P_{4}^{0}(\cos \theta)$ & $=$ & $(1 / 8)\left(35 \cos ^{4} \theta-30 \cos ^{2} \theta+3\right)$ \\
$P_{4}^{1}(\cos \theta)$ & $=$ & $-(5 / 2)\left(7 \cos ^{2} \theta-3\right) \cos ^{2} \theta \sin \theta$ \\
$P_{4}^{2}(\cos \theta)$ & $=$ & $(15 / 2)\left(7 \cos ^{2} \theta-1\right) \sin ^{2} \theta$ \\
$P_{4}^{3}(\cos \theta)$ & $=$ & $-105 \cos ^{2} \theta \sin ^{3} \theta$ \\
$P_{4}^{4}(\cos \theta)$ & $=$ & $105 \sin ^{4} \theta$ \\
\hline
\end{tabular}

\subsection{One-Dimensional Exhaustive Search}

Here we detail the discretization scheme used to perform the aforementioned 1D exhaustive search for the maxima. We exploit the closed-form description of the curve $(\theta(\phi), \phi)$ provided by Eq. (10) and parameterize the curve with $\phi \in[0,2 \pi)$. To achieve a constant spatial resolution $\Delta s=\sqrt{\Delta \theta^{2}+\sin ^{2} \theta \Delta \phi^{2}}$, we need a variable step size $\Delta \phi$ :

$$
\Delta \phi=\frac{\Delta s}{\sqrt{\theta^{\prime 2}(\phi)+\sin ^{2} \theta(\phi)}}=\frac{1+t^{2}(\phi)}{\sqrt{t^{\prime 2}(\phi)+t^{2}(\phi)+t^{4}(\phi)}} \Delta s,
$$

which is rewritten as a function of $t(\phi):=\tan \theta(\phi)$. For every $\phi$, Eq. (10) results in one, two or three real values for $t(\phi)$, for each of which $t^{\prime}(\phi)$ can be computed simply by deriving Eq. (10) with respect to $\phi$, and substituting for $\phi$ and $t$. Therefore, at each step we choose $\Delta \phi$ to be the minimum of the three (or fewer) values obtained from Eq. (11).

Next, we keep all the candidate points satisfying inequalities (5), (6), and the following, which is a relaxation of Eq. (3),

$$
\left|\psi_{\theta}(\theta, \phi)\right|<\alpha \text {. }
$$

We found an appropriate value of $\alpha=0.02 \sim 0.03$ for the threshold. Note again that inequalities (5), (6), and (12) can all be computed analytically using equations (1), (2), (8), and Table 1. The ODF maxima are then computed as the mean directions 


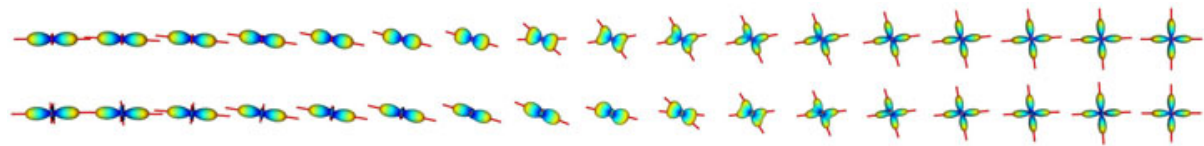

Fig. 2. Extracted maxima from synthetic ODFs with fiber crossing, in noise-free case (top), and with SNR=40 (bottom)

corresponding to the clusters of points, created by processing all the candidate points, as follows: Each point is added to a previous cluster if its Euclidean distance to the representative (mean) point of that cluster is minimum among all other clusters and is smaller than a threshold ( 0.4 was used here). If no such cluster is found, a new cluster is created, and the algorithm goes on until all the candidate points are processed.

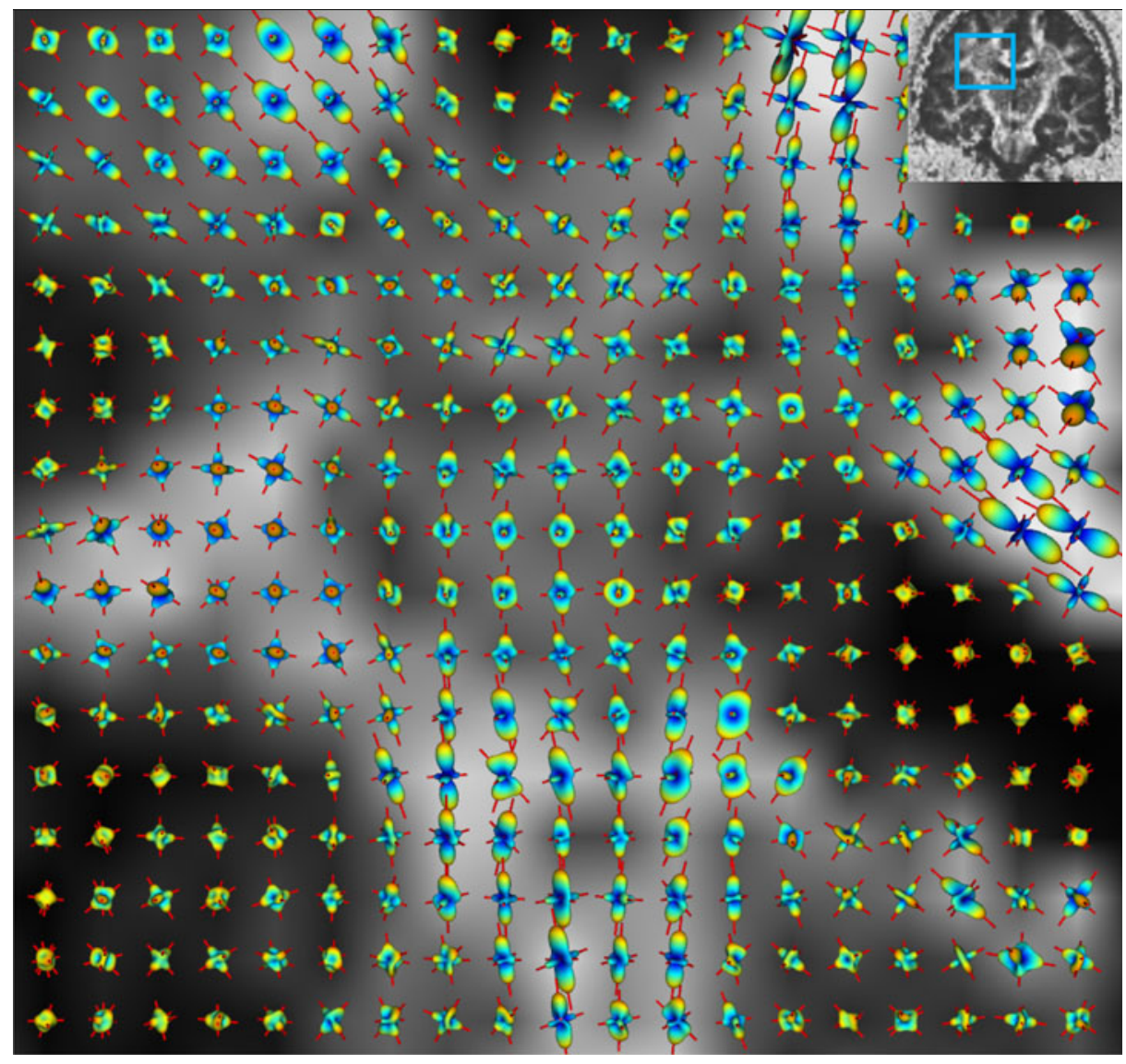

Fig. 3. Experimental results on human brain data, superimposed on the FA map 


\section{Results and Discussion}

To validate our approach, we first show results on artificial data. We simulated fiber crossing by generating diffusion images from the sum of two exponentials, $S(\hat{u})=$ $\left(e^{-\widehat{u}^{T} D_{1} \widehat{u}}+e^{-\widehat{u}^{T} D_{2} \widehat{u}}\right) / 2$, where $D_{1}$ is a diagonal matrix with diagonal entries $(9,2,2)$, and $D_{2}$ is $D_{1}$ rotated about the $z$-axis by a varying angle. CSA-ODFs were reconstructed in the fourth order RSSH basis from 76 diffusion directions, uniformly sampled on the sphere. The maxima were then extracted using the proposed technique, and results are depicted in Fig. 2 (top). Increasing the angular precision to $0.5^{\circ}$ revealed that multiple fiber orientations are resolved starting at the crossing angle of $37.5^{\circ}$. Choosing a spatial resolution of $\Delta s=0.001$, required the evaluation of the ODF at $7.7 \times 10^{4}$ points, whereas a $2 \mathrm{D}$ search on the sphere with the same resolution would cost $1.6 \times 10^{7}$ operations. When we repeated the experiment by adding Rician noise with a signal-to-noise ratio (SNR) of 40 (Fig. 2, bottom), the minimum angle where crossing was detected increased to $48^{\circ}$. Such experiments are commonly employed to evaluate the robustness of the ODF reconstruction algorithm to noise.

We also tested our method on a popular public human brain dataset [17]. CSAODFs were reconstructed in the fourth order RSSH basis from 200 diffusion images acquired at $b=3000 \mathrm{~s} / \mathrm{mm}^{2}$. Figure 3 illustrates the ODFs with their extracted maxima superimposed on the fractional anisotropy (FA) map, in the region of the centrum semiovale, where three major fiber bundles intersect. To demonstrate the performance of the proposed technique, all the maxima are shown here, including those corresponding to slight variations in the ODF (for example due to noise). Major ODF peaks corresponding to fiber orientations may however be selected by placing a threshold on the ODF [3] or on its curvature [15].

Acknowledgments. This work was partly supported by NIH (P41 RR008079, P30 NS057091, R01 EB007813, R01 MH060662, R01 EB008432, CON0000000040513014), NSF, ONR, NGA, ARO, and DARPA. We would like to thank the authors of [17] for providing us with human brain data.

\section{References}

1. Basser, P., Mattiello, J., LeBihan, D.: Estimation of the effective self-diffusion tensor from the NMR spin echo. J. Magn. Reson. B. 103(3), 247-254 (1994)

2. Tuch, D.: Q-ball imaging. Magnetic Resonance in Medicine 52(6), 1358-1372 (2004)

3. Descoteaux, M., Angelino, E., Fitzgibbons, S., Deriche, R.: Regularized, fast, and robust analytical q-ball imaging. Magnetic Resonance in Medicine 58(2), 497-510 (2007)

4. Anderson, A.: Regularized, fast, and robust analytical q-ball imaging. Magnetic Resonance in Medicine 54(5), 1194-1206 (2005)

5. Hess, C., Mukherjee, P., Han, E., Xu, D., Vigneron, D.: Q-ball reconstruction of multimodal fiber orientations using the spherical harmonic basis. Magnetic Resonance in Medicine 56(1), 104-117 (2006)

6. Aganj, I., Lenglet, C., Sapiro, G., Yacoub, E., Ugurbil, K., Harel, N.: Reconstruction of the orientation distribution function in single and multiple shell q-ball imaging within constant solid angle. Magnetic Resonance in Medicine 64(2), 554-566 (2010) 
7. Mori, S., Crain, B., Chacko, V., Van Zijl, P.: Three dimensional tracking of axonal projections in the brain by magnetic resonance imaging. Annals of Neurology 45(2), 265269 (1999)

8. Conturo, T., Lori, N., Cull, T., Akbudak, E., Akbudak, A., Shimony, J., McKinstry, R., Burton, H., Raichle, M.: Tracking neuronal fiber pathways in the living human brain. Proc. of National Academy of Sciences 96(18), 10422-10427 (1999)

9. Behrens, T., Johansen Berg, H., Jbabdi, S., Rushworth, M., Woolrich, M.: Probabilistic diffusion tractography with multiple fibre orientations: What can we gain? NeuroImage 34(1), 144-155 (2007)

10. Frey, S., Campbell, J., Pike, G., Siddiqi, K.: Dissociating the human language pathways with high angular resolution diffusion fiber tractography. Journal of Neuroscince 28(45), 11435-11444 (2008)

11. Berman, J., Chung, S., Mukherjee, P., Hess, C., Han, E., Henry, R.: Probabilistic streamline q-ball tractography using the residual bootstrap. NeuroImage 39(1), 215-222 (2008)

12. Tournier, J., Calamante, F., Gadian, D., Connelly, A.: Direct estimation of the fiber orientation density function from diffusion-weighted MRI data using spherical deconvolution. NeuroImage 23(3), 1176-1185 (2004)

13. Jansons, K., Alexander, D.: Persistent angular structure: new insights from diffusion magnetic resonance imaging data. Inverse Problems 19, 1031-1046 (2003)

14. Ghosh, A., Tsigaridas, E., Descoteaux, M., Comon, P., Mourrain, B., Deriche, R.: A polynomial based approach to extract the maxima of an antipodally symmetric spherical function and its application to extract fiber directions from the Orientation Distribution Function in Diffusion MRI. In: Metaxas, D., Axel, L., Fichtinger, G., Székely, G. (eds.) MICCAI 2008, Part II. LNCS, vol. 5242. Springer, Heidelberg (2008)

15. Bloy, L., Verma, R.: On computing the underlying fiber directions from the diffusion orientation distribution function. In: Metaxas, D., Axel, L., Fichtinger, G., Székely, G. (eds.) MICCAI 2008, Part I. LNCS, vol. 5241, pp. 1-8. Springer, Heidelberg (2008)

16. Qi, L., Han, D., Wu, E.: Principal invariants and inherent parameters of diffusion kurtosis tensors. Journal of Mathematical Analysis and Applications 349(1), 165-180 (2009)

17. Poupon, C., Poupon, F., Allirol, L., Mangin, J.: A database dedicated to anatomofunctional study of human brain connectivity. In: Proc. of the 12th Annual Meeting of OHBM (2006) 